\title{
Microbiological Pattern and Antibiotic Sensitivity in Diabetic Foot Ulcer in a Tertiary Care Center- A Cross Sectional Study
}

\author{
Authors \\ Dr Sreenivasan $M^{1}$, Dr Vinu C. ${ }^{2^{*}}$, Dr Suresh Kumar $V^{3}$ \\ ${ }^{1}$ Senior Resident, General Surgery, Govt. Medical College, Trivandrum \\ ${ }^{2}$ Associate Professor, Dept of Cardiovascular and Thoracic Surgery, Govt. Medical College, Trivandrum \\ ${ }^{3}$ Professor, Department of Cardiovascular and Thoracic Surgery, Govt Medical College, Trivandrum \\ Corresponding Author
}

Dr Vinu.C.V

Associate Professor, Dept of Cardiovascular and Thoracic Surgery, Govt. Medical College, Trivandrum

\begin{abstract}
The problem of Diabetes is rapidly growing worldwide, making it the most challenging health problem of present century. The burden is supposed to rise from 382 million adults in 2013 to 592 million adults by 2035.The organism associated with diabetic foot ulcer varies in different geographical location. The aim of the present study is to describe the pattern of microbiological spectrum in the diabetic foot ulcers. In addition, we aimed to compare the difference in various variables between the mono microbial and microbial infections in the diabetic foot ulcers and associate these with different grades of the SINBAD grading.

After a priori sample size calculation was done, 100 consecutive patients were recruited for the study. All diabetic foot ulcer patients aged more than 30 years were included in the study. Data analysis was done using $R$ statistical software. Continuous data was summarized with mean ( \pm ) and standard deviation or median and interquartile range. Categorical data were summarized with proportion and \%cent ages. Difference in the mean $( \pm) s$ were analyzed with $t$ test and Mann Whitney test. Chi square test was used to test categorical variables.

Using a pretested well-structured data collection form, patient's data were taken. All variables needed for the SINBAD grading were recorded. Sixty four percent of the study population were males. The average age of the patients was 58.1 years $(S D=9.59)$. The median duration of diabetes was 10 years $(I Q R$ 5.75-15). The most common site affected was mid foot/hind foot. Most of the lesions (48\%) occurred spontaneously, followed by trauma in $44 \%$ of the patient (Table1.) In addition, there were statistically significant difference in ulcer area when ulcer characteristics are compared across the type of microbial organisms obtained as per culture and sensitivity

In the 91 patients with infection, gram positive organism was present in 35(38.5\%) of the patients, mixed organisms in $32(35.2 \%)$ and gram negative in 24(26,4\%) of the patients. Proteus, citrobacter and klebsiella were the most common organisms in the mixed category. Ploy microbial organism constituted $39 \%$ of the patients and $61 \%$ mono microbial. Most sensitive antibiotic in our study is Gentamycin (24\%) followed by Cloxacillin(17\%) and Amikacin (16\%). Moreover, mono microbial group was sensitive to these antibiotics. Our analysis shows that an FBS > $200 \mathrm{mg} / \mathrm{dl}$ is an important factor for development of foot ulcer.Males are more affected than females. Both aerobic \& anaerobic pathogens are involved in diabetic wound Infection, but among them staph aureus is more common. Common site of ulcer in foot was mid/hind foot, developing spontaneously in a background of neuropathy \& vasculopathy.

The first requirement in the management of DFU is strict control of diabetes, \& early detection and treatment of lesions; after identifying the organism \& its antibiotic sensitivity. Regular foot care is also important. The importance of grading ulcer\& treating with appropriate antibiotic according to culture \& sensitivity is essential for controlling infection and progression of ulcer and thereby preventing limb amputation.
\end{abstract}




\section{Introduction}

The problem of Diabetes is rapidly growing worldwide, making it the most challenging health problem of the present century. The number of diabetics is supposed to rise from 382 million adults in 2013 to 592 million adults by 2035, and is further aggravated by changing population demographics, urbanization, and lifestyle factors ${ }^{1}$ Prevalence of DFU is estimated to be $4-10 \%$ of total diabetics, with a life time risk to develop DFU is 25\%). Foot ulceration increases the risk of lower limb amputation. Almost $85 \%$ of amputation is preceded by foot ulceration that later progress to spreading infection or gangrene. DFU affects both physical and mental health of patient leading to poor quality of $\operatorname{life}^{2}$, Moreover, this imposes a substantial economic burden, where cost of hospitalization and amputation account for more than $50 \%$ of money spent ${ }^{3}$

Of the various causes of diabetic foot ulcers, infection contributes to an important role. Ulcer is not directly due to infection ${ }^{2}$, but once an ulcer is complicated by infection, the risk for amputation increases; especially in ischaemic and neuroischaemic ulcers. Diabetic neuropathy, which occur in $25 \%$ of diabetic patients; in the presence of hyperglycemia, with or without significant vascular impairment, can form a bed for the entry of bacterial pathogens and the progression of the infection. In the present scenario, foot infection is the most common diabetic complication leading on to hospitalization and lower limb amputations 4, , 6,7.

Various grading and classification system are used to categorize and prognosticate the diabetic ulcers. There is no well accepted classification system for diabetic foot ulcers. A clinical classification should be relatively flexible and descriptive, but the one used for auditing must be more structured and simple enough for use in larger populations. One used for prospective research should be selective and exclusive. To compare outcomes among different countries, a new scoring system was developed and- the "SINBAD "scoring system; which is a clinical classification that includes various factors important in the healing of a diabetic ulcer.

The organism associated with diabetic foot ulcer varies in different geographical location. There is a paucity of studies that elucidate the microbiological spectrum in diabetic foot ulcers in various SINBAD grades. The aim of the present study is to describe the pattern of microbiological spectrum in the diabetic foot ulcers. In addition, we aimed to compare the difference in various variables between the mono microbial and poly microbial infections in the diabetic foot ulcers and associate these with different grades of the SINBAD grading.

\section{Materials and Methods}

We conducted this hospital-based cross-sectional study at the department of surgery medical college, Trivandrum. Ethics committee approval was obtained before starting the study. A formal priori sample size calculation was done. Thereafter, 100 consecutive patients were recruited for the study. All diabetic foot ulcer patients aged more than 30 years were included in the study. Vascular disorders like vasculitis and neurological disorders like leprosy were excluded from the study. Those patients with co existing renal diseases were excluded from the study.

Using a pretested well-structured data collection form, patient's data were taken. All variables needed for the SINBAD grading were recorded. Detailed history and clinical examination were done in all patients. Specimens (pus, wound exudates, tissue biopsy) for microbiological studies were obtained from the ulcer region. Pus and exudates were collected from the margins and the base of the ulcer using a sterile swab stick, which was then transported in a clean and sterile test tube soon after collection. Tissue biopsy was taken with a sterile blade/knife in wedge shape, including base and margin of ulcer along with wound swabs from the same site and was then transported in sterile solution of normal saline and sterile test tube respectively. These specimens were immediately transported to the microbiology laboratory and identification of microorganisms 
were done according to the standard microbiological procedures.

Data analysis was done using $\mathrm{R}$ statistical software. Continuous data was summarized with mean and standard deviation or median and interquartile range. Categorical data were summarized with proportion and percentage. Differences in the means were analyzed with t-test and Mann-Whitney test. Chi-square test was used to test categorical variables.

\section{Results}

In this study, we enrolled 100 diabetic patients with foot ulcers. Table 1 shows the baseline demographic features of the patients. Sixty-four percent of the study population were males. The average age of the patients was 58.1 years $(\mathrm{SD}=9.59)$. The median duration of diabetes was 10 years (IQR 5.75-15). The most common site affected was mid foot/hind foot. Most of the lesions $(48 \%)$ occurred spontaneously, followed by trauma in $44 \%$ of the patient (Table1.)
Table 1 Baseline characteristics of 100 patients with diabetic foot ulcer

\begin{tabular}{ll} 
& {$[$ ALL] N=100 } \\
\hline Age & $58.1(9.59)$ \\
Sex: & $64(64.0 \%)$ \\
Male & $36(36.0 \%)$ \\
Female & $1(1.00 \%)$ \\
Type of DM: & $99(99.0 \%)$ \\
I & $10.0[5.75 ; 15.0]$ \\
II & \\
Duration of DM & $40(40.0 \%)$ \\
Treatment: & $60(60.0 \%)$ \\
Irregular & \\
Regular & $56(56.0 \%)$ \\
Medication: & $14(14.0 \%)$ \\
OHA & $30(30.0 \%)$ \\
Insulin & \\
OHA plus insulin & $66(66.0 \%)$ \\
Smoker: & $34(34.0 \%)$ \\
Non smoker & \\
Smoker & $65(65.0 \%)$ \\
Alcoholic: & $35(35.0 \%)$ \\
Non alcoholic & \\
Alcoholic & $29(29.0 \%)$ \\
FBS: & $40(40.0 \%)$ \\
$70-110$ & $31(31.0 \%)$ \\
110-200 &
\end{tabular}

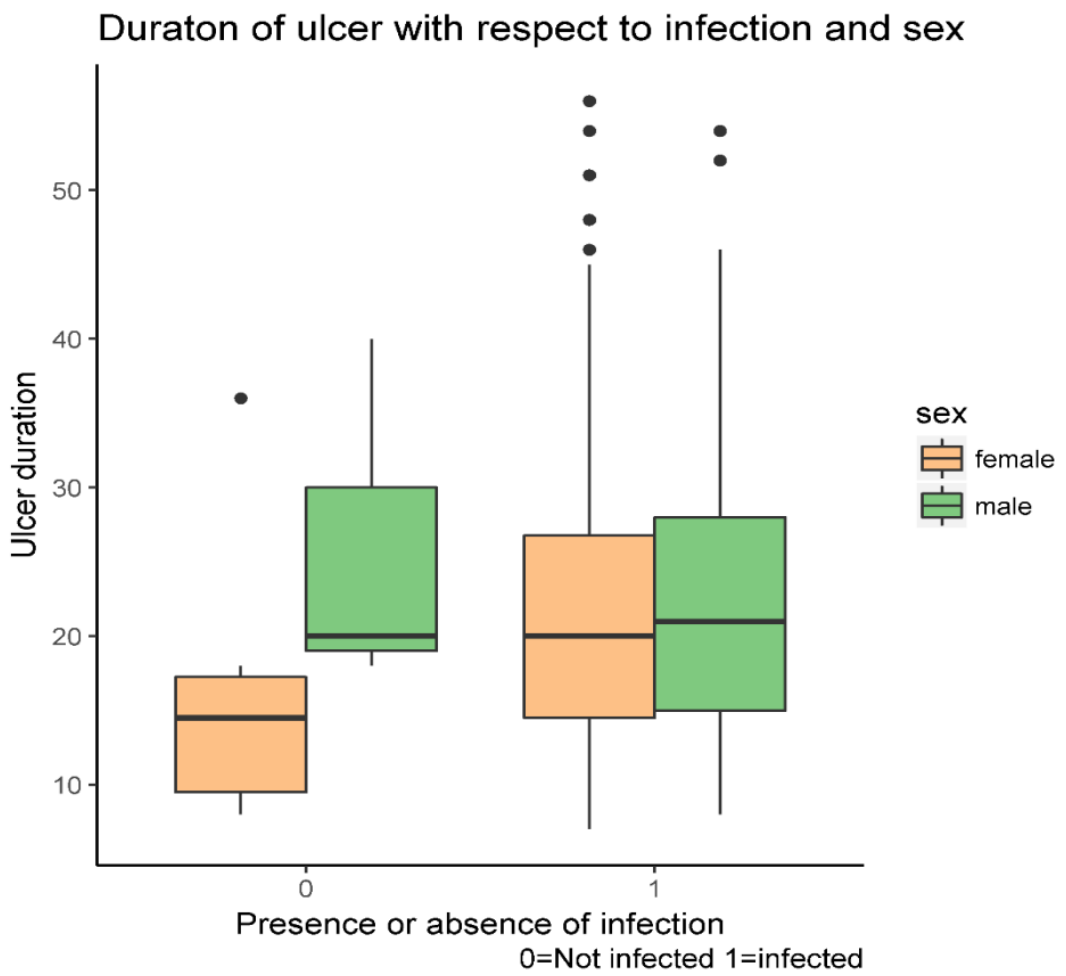

The details of the patients with microbiological spectrum and culture positivity are summarized in table2. Comparison of various baseline features across monomicrobial and ploy microbial organisms shows statistically significant difference only in duration of diabetes mellitus (figure 1 and 2 ). 
Table 2: comparison of baseline features across monomicrobial vesus poly microbial

\begin{tabular}{|c|c|c|c|c|}
\hline & [ALL] $N=100$ & Monomicrobial N=61 & Polymicrobial N=39 & p.overall \\
\hline Age & $58.1(9.59)$ & $59.4(10.5)$ & $56.2(7.79)$ & 0.085 \\
\hline Sex: & & & & 0.293 \\
\hline Male & $64(64.0 \%)$ & $42(68.9 \%)$ & $22(56.4 \%)$ & \\
\hline Female & $36(36.0 \%)$ & $19(31.1 \%)$ & $17(43.6 \%)$ & \\
\hline Type of DM: & & & & 1.000 \\
\hline I & $1(1.00 \%)$ & $1(1.64 \%)$ & $0(0.00 \%)$ & \\
\hline II & $99(99.0 \%)$ & $60(98.4 \%)$ & $39(100 \%)$ & \\
\hline Duration & $10.0[5.75 ; 15.0]$ & $12.0[7.00 ; 16.0]$ & $8.00[3.00 ; 12.0]$ & 0.007 \\
\hline Treatment & & & & 0.706 \\
\hline Irregular & $40(40.0 \%)$ & $23(37.7 \%)$ & $17(43.6 \%)$ & \\
\hline Regular & $60(60.0 \%)$ & $38(62.3 \%)$ & $22(56.4 \%)$ & \\
\hline Medication: & & & & 0.664 \\
\hline OHA & $56(56.0 \%)$ & $32(52.5 \%)$ & $24(61.5 \%)$ & \\
\hline Insulin & $14(14.0 \%)$ & $9(14.8 \%)$ & $5(12.8 \%)$ & \\
\hline Both & $30(30.0 \%)$ & $20(32.8 \%)$ & $10(25.6 \%)$ & \\
\hline Smoker: & & & & 0.742 \\
\hline No & $66(66.0 \%)$ & $39(63.9 \%)$ & $27(69.2 \%)$ & \\
\hline Yes & $34(34.0 \%)$ & $22(36.1 \%)$ & $12(30.8 \%)$ & \\
\hline Alcoholic: & & & & 0.074 \\
\hline No & $65(65.0 \%)$ & $35(57.4 \%)$ & $30(76.9 \%)$ & \\
\hline Yes & $35(35.0 \%)$ & $26(42.6 \%)$ & $9(23.1 \%)$ & \\
\hline FBS: & & & & 0.546 \\
\hline 0 to 110 & $29(29.0 \%)$ & $18(29.5 \%)$ & $11(28.2 \%)$ & \\
\hline $110-200$ & $40(40.0 \%)$ & $22(36.1 \%)$ & $18(46.2 \%)$ & \\
\hline$>200$ & $31(31.0 \%)$ & $21(34.4 \%)$ & $10(25.6 \%)$ & \\
\hline
\end{tabular}

Figure 2

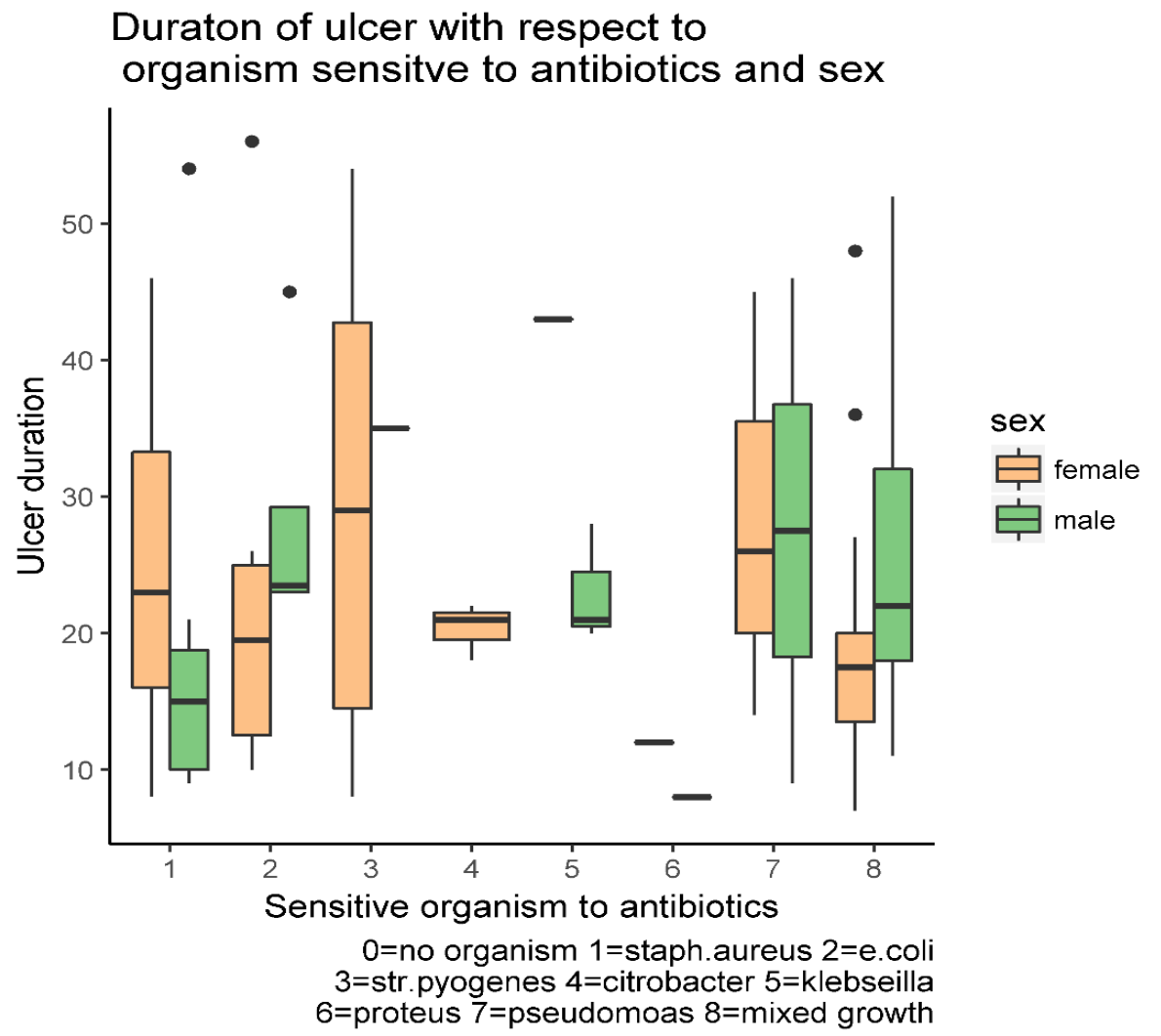

In addition, there was statistically significant difference in ulcer area when ulcer characteristics are compared across the type of microbial organisms obtained as per culture and sensitivity (table 3) (figure3). 
Table 3: comparison of features of ulcers in mono microbial versus Polymicrobial.

\begin{tabular}{lcccc} 
& {$[\mathrm{ALL}] \mathrm{N}=100$} & Monomicrobial N=61 & Polymicrobial N=39 & p.overall \\
\hline Ulcer duration & $20.0[14.0 ; 27.2]$ & $22.0[14.0 ; 34.0]$ & $18.0[14.5 ; 24.0]$ & 0.233 \\
Area & $9.00[6.00 ; 14.2]$ & $12.0[6.00 ; 15.0]$ & $6.00[4.00 ; 10.0]$ & 0.024 \\
Mode : & & & & 0.285 \\
$\quad$ Trauma & $44(44.0 \%)$ & $24(39.3 \%)$ & $20(51.3 \%)$ & \\
$\quad$ Spontaneous & $48(48.0 \%)$ & $33(54.1 \%)$ & $15(38.5 \%)$ & \\
$\quad$ Weight bear & $7(7.00 \%)$ & $4(6.56 \%)$ & $3(7.69 \%)$ & \\
$\quad$ Injury & $1(1.00 \%)$ & $0(0.00 \%)$ & $1(2.56 \%)$ & 1.000 \\
Site: & & & & \\
$\quad$ Forefoot & $22(22.0 \%)$ & $13(21.3 \%)$ & $9(23.1 \%)$ & \\
$\quad$ Mid/hindfoot & $78(78.0 \%)$ & $48(78.7 \%)$ & $30(76.9 \%)$ & \multirow{2}{*}{0.296} \\
Ulcer area: & & & & \\
$\quad$ Less than 1cm & $4(4.00 \%)$ & $1(1.64 \%)$ & $3(7.69 \%)$ & \\
$\quad$ More than 1cm & $96(96.0 \%)$ & $60(98.4 \%)$ & $36(92.3 \%)$ & \multirow{2}{*}{1.000} \\
Depth: & & & & \\
$\quad$ superficial & $5(5.00 \%)$ & $3(4.92 \%)$ & $2(5.13 \%)$ & \\
$\quad$ Deep & $95(95.0 \%)$ & $58(95.1 \%)$ & $37(94.9 \%)$ &
\end{tabular}

Figure 3

Duraton of ulcer with respect to ulcer ara and sex

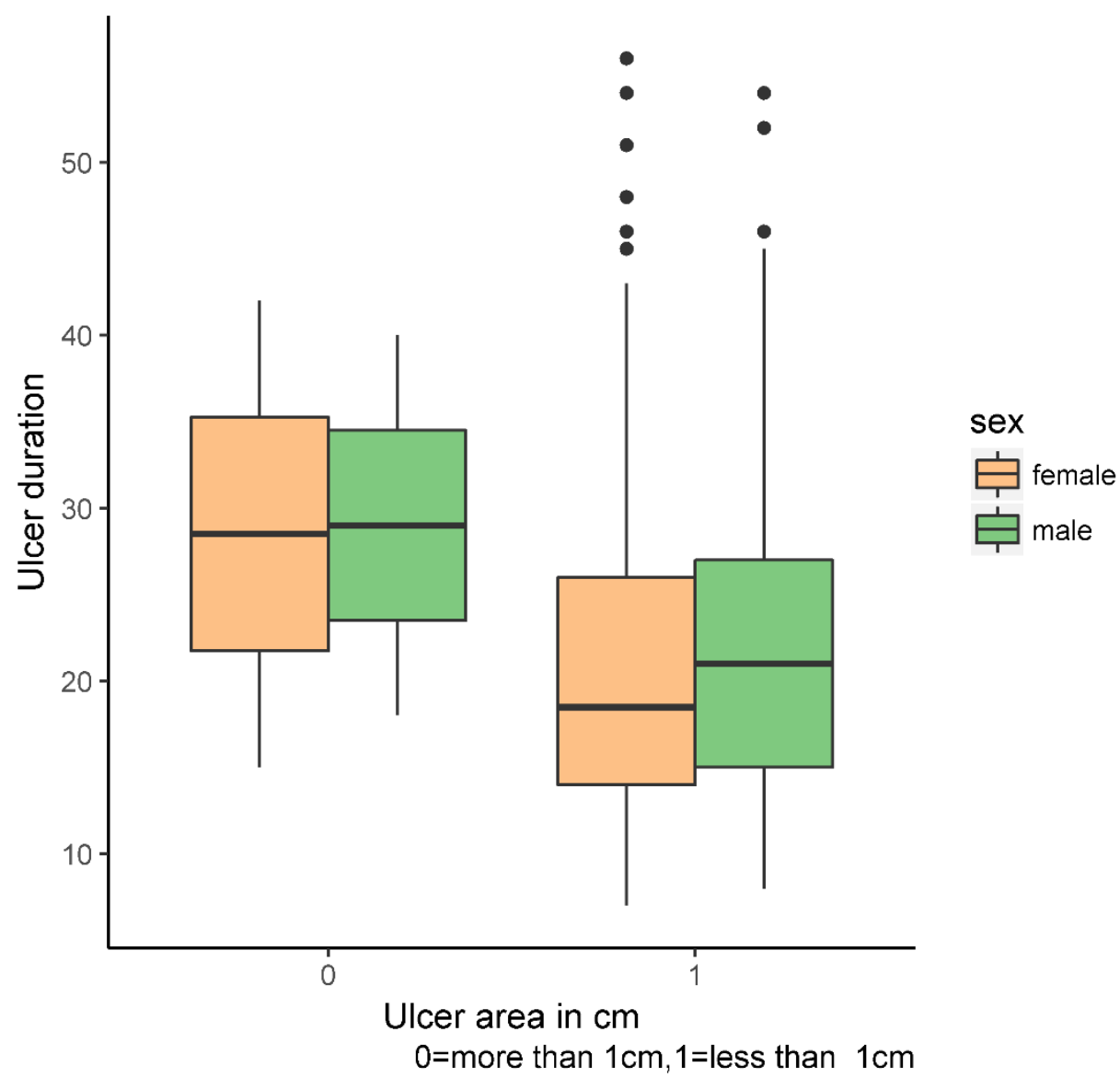

In the 91 patients with infection, gram-positive organism was present in $35(38.5 \%)$ of the patients, mixed organisms in $32(35.2 \%)$ and gram negative in $24(26.4 \%) 4 \%$ of the patients. Proteus, Citrobacter, and Klebsiella were the most common organisms. Poly microbial organism constituted $39 \%$ of the patients and $61 \%$ mono microbial.

Sensitivity of the microorganisms to various antibiotics are given in table4. 
Table 4: Antimicrobial sensitivity of different organisms

\begin{tabular}{|c|c|c|c|c|}
\hline & [ALL] $N=100$ & Monomicrobial N=61 & Polymicrobial N=39 & p.overall \\
\hline Pencilllin : & & & & 0.154 \\
\hline Sensitive & $4(4.00 \%)$ & $4(6.56 \%)$ & $0(0.00 \%)$ & \\
\hline Resistant & $96(96.0 \%)$ & $57(93.4 \%)$ & $39(100 \%)$ & \\
\hline Gentamycin: & & & & 0.001 \\
\hline Sensitive & $24(24.0 \%)$ & $22(36.1 \%)$ & $2(5.13 \%)$ & \\
\hline Resistant & $76(76.0 \%)$ & $39(63.9 \%)$ & $37(94.9 \%)$ & \\
\hline Piperacllin-Tazobactum: & & & & 0.153 \\
\hline Sensitive & $5(5.00 \%)$ & $5(8.20 \%)$ & $0(0.00 \%)$ & \\
\hline Resistant & $95(95.0 \%)$ & $56(91.8 \%)$ & $39(100 \%)$ & \\
\hline Amikacin : & & & & 0.008 \\
\hline Sensitive & $16(16.0 \%)$ & $15(24.6 \%)$ & $1(2.56 \%)$ & \\
\hline Resistant & $84(84.0 \%)$ & $46(75.4 \%)$ & $38(97.4 \%)$ & \\
\hline Nitrofurantoin: & & & & 0.079 \\
\hline Sensitive & $6(6.00 \%)$ & $6(9.84 \%)$ & $0(0.00 \%)$ & \\
\hline Resistant & $94(94.0 \%)$ & $55(90.2 \%)$ & $39(100 \%)$ & \\
\hline Cloxacillin: & & & & 0.537 \\
\hline Sensitive & $17(17.0 \%)$ & $12(19.7 \%)$ & $5(12.8 \%)$ & \\
\hline Resistant & $83(83.0 \%)$ & $49(80.3 \%)$ & $34(87.2 \%)$ & \\
\hline Ceftriaxone: & & & & 0.011 \\
\hline Sensitive & $9(9.00 \%)$ & $9(14.8 \%)$ & $0(0.00 \%)$ & \\
\hline Resistant & $91(91.0 \%)$ & $52(85.2 \%)$ & $39(100 \%)$ & \\
\hline
\end{tabular}

\section{Discussion}

In this study, we aimed to find out the microbiological pattern in diabetic foot ulcers in our setting, a tertiary care teaching center. Our study has shown that diabetic foot ulcers are more commonly involved with mono microbial organisms. Out of this, gram positive constitute the major part. Another important finding we observed is that there is a statistically significant difference in the area of the ulcer and duration of diabetes in the mono microbial group. There was a reduction of about $50 \%$ in the ulcer size in the ploy microbial group.

There could be multiple reasons for the drastic reduction almost by $50 \%$ in the ulcer area in the poly microbial group. The selective referral of patients with more severe ulcers may explain the increased prevalence of mono microbial infection in our group. Other factors like emergence of multidrug resistant bacteria, availability of over the counter, drugs and unscrupulous prescription of antibiotics could have resulted in the emergence of more virulent organisms. Most of the ulcers in the mono microbial group occurred spontaneously whereas trauma was the precipitating cause in most of the poly microbial group. The decreased immunity and trauma along

with multiple organisms may be the cause in the second group. In addition, the duration of the diabetes was longer in the mono microbial group. This again would have reduced the immunity more compared to the other group. Longer duration and highly virulent organism may be the contributing factors in the first group.

In many studies in the literature, poly microbial infections are more prevalent than mono microbial infections. However, in our study mono-microbial infections are more common accounting for almost $61 \%$ of culture. Poly-microbial accounted only for 39. Staphylococcus aureus (26\%) is the commonest organism in the present study. In the study conducted by Rajah et al, Staphylococcus constituted the major organisms $(41 \%)^{8}$. In a study conducted by European society of clinical microbiology and infectious disease; Helsinki Finland, $65 \%$ were gram-positive cocci, whereas Staphylococcus accounted for $42 \%$ cases $^{9}$. This is mainly because skin lesions are mostly caused by gram-positive organisms. A variety of physiological and metabolic disturbances contribute to foot ulceration in diabetes patients. Endogenous bacterium that usually colonize a wound is potentially pathogenic in diabetic ulcers. Immunological disturbance also contributes to 
pathophysiology of foot ulcer. These include abnormalities of migration, phagocytosis, intracellular killing, and chemotaxis. The cellular immune response and monocyte function are also reduced in diabetes.

Infection in diabetic foot can be aerobic or anaerobic; aerobic being the most common. The impact of anaerobes was first reported by Louie et al and subsequently by many researchers ${ }^{10}$. In the study by Edmonds et all, gram-positive cocci were the most prominent anaerobes ${ }^{11}$.Few reports are also available regarding the incidence of fungal pathogens in diabetic foot infections. As reported by Louie et al diabetic foot ulcer infection is usually poly microbial in nature ${ }^{10}$. E. coli, Streptococci \& Pseudomonas $(10 \%, 9 \%, 5 \%)$ are the figures In our study.

The most sensitive antibiotic in our study is Gentamycin (24\%) followed by Cloxacillin (17\%) and Amikacin (16\%). Moreover, mono microbial group was more sensitive to these antibiotics. In the study by Kavitha et al ${ }^{12}$, local antibiotics like gentamycin were found to be effective in reducing the antimicrobial load in diabetic foot ulcers. However, most of the other literature does not show any evidence supporting the use of any local antibiotics in diabetic foot. In addition, our analysis shows that an FBS > $200 \mathrm{mg} / \mathrm{dl}$ is an important factor for development of foot ulcer.

According to Lehto et al, the risk of amputation increases proportionately with increase in plasma glucose level ${ }^{13}$. Boyko et $\mathrm{al}^{5}$ reported an increase risk for diabetic foot ulcer with severe hypoglycemia. Chaturvedi et al reported an increased glucose level as a key risk factor for amputation $^{14}$. Many studies have shown that increased levels of blood glucose over a long period of time, as assessed by $\mathrm{HbAl} \mathrm{C}$, is a better predictor for diabetic foot amputation.

Our study has a few limitations. One of the main limitations in our study is the hospital setting. A community-based study will better represent the true nature of microbiological pattern in the society. However, our results are pertinent in high-risk patients who are referred to tertiary care centers. Other potential drawbacks in our study are the relatively low sample size and crosssectional nature of the study.

\section{Conclusion}

Diabetic foot ulcer is common in old age and is related to duration of diabetes. Males are more affected than females. Both aerobic \& anaerobic pathogens are involved in diabetic wound infection but among them, staph aureus is more common. Common site of ulcer in foot was Common site of ulcer in foot was mid/hind foot, developing spontaneously in a background of neuropathy \& vasculopathy. The first requirement in the management of DFU is strict control of diabetes, \& early detection and treatment of lesions after identifying the organism \& its antibiotic sensitivity. Regular foot care is also important. The importance grading of ulcer \& treating with appropriate antibiotic according to culture \& sensitivity is essential for controlling infection and progression of ulcer and thereby preventing limb amputation.

\section{Reference}

1. Federation ID. IDF diabetes atlas. Brussels: International Diabetes Federation 2013.

2. Intemational Working Group on the Diabetic Foot. International consensus on the diabetic foot and practical guidelines on the management and the prevention of the diabetic foot Amsterdam Intematlonal Working Group on the Diabetic Foot DVD 2011.

3. Acker K, Leger P, Haltemann A, Chawla A. Van Burden of diabetic foot disorders, guidelines for management, and disparities in implementation in Europe: a systematic literature review. Diabetes Metab Res Rev Jan 27 doi 101002dm1T2523 Epub ahead of print 2014.

4. Prompers L, Schaper N, Apelqvist J, et al. Prediction of outcome in individuals with diabetic foot ulcers: focus on the differences between individuals with and without peripheral arterial disease. The 
EURODIALE Study. Diabetologia 2008; 51(5): 747-55.

5. Boyko EJ, Ahroni JH, Stensel V, Forsberg RC, Davignon DR, Smith DG. A prospective study of risk factors for diabetic foot ulcer. The Seattle Diabetic Foot Study. Diabetes care 1999; 22(7): 1036-42.

6. Reiber GE, Vileikyte L, Boyko EJ, et al. Causal pathways for incident lowerextremity ulcers in patients with diabetes from two settings. Diabetes care 1999; 22(1): 157-62.

7. Annstrong DG, Lavery LA, Harkless LB. Validation of a diabetic wound classification system: contribution of depth, infection, and vascular disease to the risk of amputation. Diabetes Care 855 859 1998; 21 SRC - GoogleScholar.

8. Raja NS. Microbiology of diabetic foot infections in a teaching hospital in Malaysia: a retrospective study of 194 cases. J Microbiol Immunol Infect 2007; 40(1): 39-44.

9. 19th European Congress of Clinical Microbiology and Infectious Diseases.

10. Louie TJ, Bartlett JG, Tally FP, Gorbach SL. Aerobic and anaerobic bacteria in diabetic foot ulcers. Annals of internal medicine 1976; 85(4): 461-3.

11. Edmonds M. The treatment of diabetic foot infections: focus on ertapenem. Vasc Health Risk Manag 2009; 5: 949-63.

12. Kavitha KV, Tiwari S, Purandare VB, Khedkar S, Bhosale SS, Unnikrishnan AG. Choice of wound care in diabetic foot ulcer: A practical approach. World J Diabetes 2014; 5(4): 546-56.

13. Lehto S, Rönnemaa T, Pyörälä K, Laakso M. Risk factors predicting lower extremity amputations in patients with NIDDM. Diabetes care 1996; 19(6): 607-12.

14. Chaturvedi N, Stevens LK, Fuller JH, Lee ET, Lu M. Risk factors, ethnic differences and mortality associated with lowerextremity gangrene and amputation in diabetes. The WHO Multinational Study of Vascular Disease in Diabetes. Diabetologia 2001; 44 Suppl 2: S65-71. 